IAU Colloquium 164: Radio Emission from Galactic and Extragalactic Compact Sources

ASP Conference Series, Vol. 144, 1998

J. A. Zensus, G. B. Taylor, \& J. M. Wrobel (eds.)

\title{
Radio Properties of the X-Ray Binary GX 339-4
}

R. K. Sood

Australian Defence Force Academy, UNSW, Canberra, Australia

D. Campbell-Wilson

School of Physics, University of Sydney, Sydney, Australia

Ph. Durouchoux \& S. Corbel

C.E. Saclay, DAPNIA, Service d'Astrophysique, France

R. Fender

Astronomy Centre, University of Sussex, Brighton, UK

R. Spencer

University of Manchester, Jodrell Bank, Cheshire, UK

M. A. Nowak

JILA, Colorado, USA

A. K. Tzioumis

Australia Telescope National Facility, Epping, Australia

\begin{abstract}
GX 339-4 has been monitored over a period of two and a half years with MOST and ATCA to investigate the flux variations and spatial structure of its radio emission. The data are summarized and discussed.
\end{abstract}

The low mass X-ray binary GX 339-4 exhibits four distinct X-ray states that makes its behavior similar to Cyg X-1 (Nowak 1994). Radio emission was discovered from it in 1994 (Sood et al. 1997), and has since been frequently monitored and mapped. The observations were made between 1994 May 25 and 1997 Feb 18 with MOST (Molonglo Observatory Synthesis Telescope) at $843 \mathrm{MHz}$ and ATCA (Australia Telescope Compact Array) at higher frequencies up to 8640 $\mathrm{MHz}$. The flux level at these frequencies has fluctuated with a highest value of $\sim 12 \mathrm{mJy}$. On two occasions, TJD 10196 and TJD 10247, the source was below the $1 \sigma$ upper limit values of 0.7 and $1.0 \mathrm{mJy}$ respectively, at $843 \mathrm{MHz}$. There are variations in intensity of $\sim 50 \%$ on the timescale of one week.

Spectral data obtained on $1997 \mathrm{Feb} 18$ are shown in Figure 1. The optically thick, relatively flat spectrum does not show significant change compared to the spectrum determined 7 months earlier (Fender et al. 1997). Indeed, measurements over two years, do not indicate deviations from this shape. Further simultaneous observations at a number of radio wavelengths are essential to study the possible evolution of the spectrum, and hence the nature of the emission region.

Radio maps were made at ATCA on 1994 Nov 14, 1995 Sep 3, 1996 Jul 11-14, and 1997 Feb 3, 10, 17. GX 339-4 exhibits a point source structure on all days except as follows. An extended feature at $8640 \mathrm{MHz}$ was signficant at the $15 \sigma$ level on $1996 \mathrm{Jul} 11-14$, and at the $6 \sigma$ level on 1997 Feb 3. However, distortions due to atmospheric phase errors cannot be ruled out. There is no clear evidence for radio jets over two years of observations. The source was in a low state for all our observations, as determined by BATSE and XTE ASM light curves. 
A number of properties of GX 339-4 show close similarity with those of the other strong black hole ( $\mathrm{BH})$ candidate, $\mathrm{Cyg} \mathrm{X}-1$. They both exhibit an absence of radio bursts, and an absence of extended radio jet structure. The radio spectra are flat. The X-ray emission has a hard tail and exhibits rapid variability. There is additional evidence for long term X-ray variability. Cyg X-1 has a 10 month cycle, while GX 339-4 exhibits a 14.5 month cycle (Durouchoux et al. 1997). The reported orbital periods are also short ( $5.6 \mathrm{~d}$ and $14.8 \mathrm{~h}$ respectively). However, there are important differences. Cyg X-1 is an HMXRB in which a radio jet could be disrupted by radiation pressure from the supergiant companion. In the LMXRB GX 339-4, such an instability should not occur. There are no consistent similarities with the radio properties of other $\mathrm{BH}$ radio $\mathrm{X}$-ray binaries (Hjellming \& Han 1995, Fender, Bell-Burnell, \& Waltman 1997).

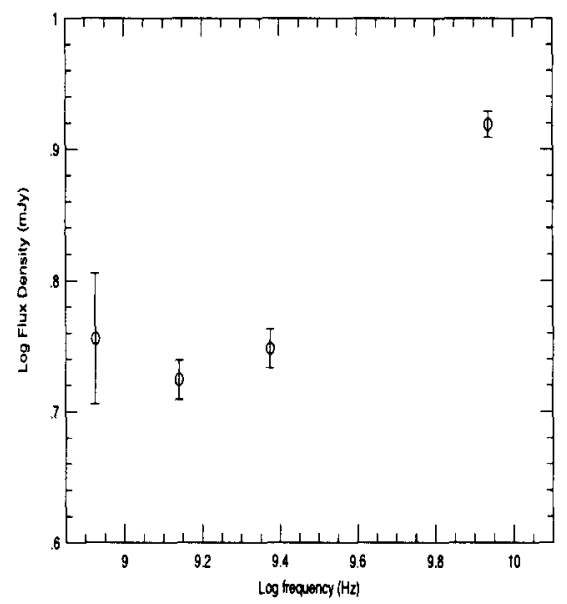

Figure 1. Radio Spectrum of GX 339-4 on 1997 Feb 18.

Acknowledgments. MOST is operated by the University of Sydney and funded by grants from the Australian Research Council. We thank the director and staft of the Australia Telescope National Facility for the use of the ATCA.

\section{References}

Durouchoux, Ph., et al. 1997. ApJ, submitted.

Fender, R. P., Bell-Burnell, S. J., \& Waltman, E. B. 1997. Vistas in Astron, 41, 3.

Fender, R. P., et al. 1997. MNRAS, 286, L29.

Hjellming, R. M. \& Han, X. 1995. in Radio Properties of X-ray Binaries, (CUP), 308.

Nowak, M. A. 1994. $A p J, 422,688$.

Sood, R. K., et al. 1997. in The Transparent Universe, ESA SP-382, 201. 